\title{
Stereo block copolymers of $\mathrm{L}$ - and $\mathrm{D}$-lactides
}

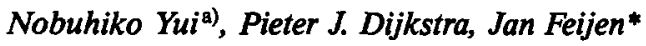 \\ Department of Chemical Technology, University of Twente P.O. Box 217, 7500 AE Enschede, \\ The Netherlands
}

(Date of receipt: August 29, 1989)

\section{SUMMARY:}

Sequential diblock copolymers composed of $\mathbf{t}$ - and D-lactic acid residues were synthesized through a living ring-opening polymerization of $\mathrm{L}$ - and D-lactide initiated by aluminium tris(2-propanolate). The composition of the block copolymers was varied by changing the reaction conditions and monomer over initiator ratio and confirmed by ${ }^{1} \mathrm{H}$ NMR analysis, molecular weight determination and optical rotation measurements. Molecular weights ranged from 1,3 to $2,0 \cdot 10^{4}$ with $1,2<\bar{M}_{\mathrm{w}} / \bar{M}_{\mathrm{n}}<1,4$. Stereocomplex formation in all block copolymers was determined using differential scanning calorimetry showing melting temperatures of about $205^{\circ} \mathrm{C}$.

\section{Introduction}

Based on their good biodegradation properties, biocompatibility, high mechanical strength, and excellent shaping and moulding properties, poly(lactide)s have been frequently applied as implantable carriers for drug delivery systems as well as surgical repair materials ${ }^{1-8)}$.

Poly(lactide) exists in several enantiomeric forms, $1, \mathrm{D}, \mathrm{DL}$, and meso ${ }^{7}$. The polymers derived from the $\mathrm{L}$ - and $\mathrm{D}$-monomers are semi-crystalline, while those from the $\mathrm{DI}$ (racemic mixture of $\mathrm{L}$ and $\mathrm{D}$ ) monomers are amorphous. Recently, it has been reported that blending of poly(I-lactide) and poly(D-lactide) gives rise to the formation of a stereocomplex (polyracemate complex) which shows a crystalline structure and morphology different from the pure polymers ${ }^{9,10)}$. A complex from poly(I-lactide) and poly(D-lactide) was obtained by pouring a solution of these polymers in dichloromethane into an excess of methanol. The complex shows a melting temperature which is approximately $50^{\circ} \mathrm{C}$ higher than that of poly(L-lactide) and poly(D-lactide). The formation of such stereocomplexes has also been reported for blends of several isotactic polymers. For instance, Prud'homme et al. ${ }^{11)}$ reported the formation of a stereocomplex between enantiomeric poly ( $\alpha$-methyl- $a$-ethyl- $\beta$-propiolactone)s, which has a melting point roughly $40^{\circ} \mathrm{C}$ higher than that of the isotactic polymers. This complex is formed preferentially between the two enantiomeric polymers. The morphology of the sample, even if this includes an excess of one of the enantiomeric polymers, is mainly controlled by the stereocomplex formation.

Stereocomplex formation is also expected to occur in the case of block copolymers of $\mathrm{L}$ - and D-lactides. Furthermore, the degree of stereocomplex formation may be controlled by the composition of the block copolymers. Such complexes may have a) Current address: Institute of Biomedical Engineering, Tokyo Women's Medical College, 8-1
Kawada, Shinjuku-ku, Tokyo 162, Japan. 
different mechanical properties and may show rates of degradation different from those of the homopolymers and the stereocomplex of the homopolymers. Previously, Teyssié et al. ${ }^{12,13)}$ and Kricheldorf et al. ${ }^{14,15)}$ have demonstrated that the polymerization of lactones by aluminium tris(2-propanolate) (1) occurs through an anionic-type coordinated insertion of the lactone into the aluminium-isopropoxy bond, involving an acyl-oxygen cleavage of the lactone, and that the propagation step proceeds through a "living" polymerization mechanism without any chain transfer or termination reactions. This would allow us to synthesize sequential diblock-copolymers of $\mathbf{L}$ - and D-lactides with a narrow molecular weight distribution and well defined block-lengths.

$\mathrm{Al}\left[\mathrm{OCH}\left(\mathrm{CH}_{3}\right)_{2}\right]_{3}$

This paper reports on the synthesis and characterization of sequential diblock copolymers of poly(L-lactide) and poly(D-lactide), obtained by a living ring-opening polymerization of $\mathrm{L}$ - and $\mathrm{D}$-lactides, initiated by aluminium tris(2-propanolate) (1).

\section{Experimental part}

Reagents: $\mathrm{L}(-)$-lactide (I-LA), and D(+)-lactide(D-LA) from CCA Biochem. b. v., Gorinchem, the Netherlands) were purified by recrystallization from distilled toluene under a nitrogen atmosphere and then dried for $24 \mathrm{~h}$ i. vac. Aluminium tris(2-propanolate) (synthetic grade; from Merck, Darmstadt, West Germany) was dried i. vac. for at least $2 \mathrm{~h}$ just before use.

Block copolymerization of $\mathrm{L}-$ and $\mathrm{D}-\mathrm{LA}$ : The block copolymerization of $\mathrm{L}$ - and D-LA was carried out in a round-bottomed flask equipped with a condenser, a three way cock with a rubber septum, a dropping funnel for addition of D-LA solution, and stirred magnetically. The system was previously dried $i$. vac. for at least $5 \mathrm{~h}$ and purged with argon. $\mathrm{L}$ - and D-LA were weighed into the flask and the funnel, respectively, and dried i. vac. for at least $5 \mathrm{~h}$. The required amounts of distilled toluene to dissolve $\mathrm{L}$ - and D-LA were introduced through the rubber septum into the flask and the funnel, respectively. Then, $0,05 \mathrm{~mol} / 1$ aluminium tris(2-propanolate) (1) solution in toluene was added to the flask through the rubber septum under an argon atmosphere. The system was kept at $90^{\circ} \mathrm{C}$ in a thermostated bath for the necessary period of time. As soon as the I-LA was completely polymerized, the D-LA solution in toluene was added dropwise. In order to examine monomer conversions in the first step (L-LA) and the second step (D-LA) polymerization, samples were taken from the reaction mixture at different time periods. The conversions of the block-copolymerization reactions were followed by ${ }^{1} \mathrm{H}$ NMR spectroscopy of the samples in $\mathrm{CDCl}_{3}$. The NMR spectra were recorded on a Nicolet $200 \mathrm{MHz}$ NMR apparatus using TMS as internal reference. The percentages of monomer conversions in the crude products were determined from the relative intensities of the methyl proton doublets of monomer $(\delta=$ $1,63-1,72)$ and polymer $(\delta=1,53-1,63)^{16)}$. After completion of the reaction, the reaction mixture was poured into an excess of methanol to precipitate the polymer. The polymer was dried i. vac. for at least $24 \mathrm{~h}$.

Characterization of the block copolymer: The number-average degree of polymerization $\left(\overline{\mathbf{D P}}_{\mathrm{n}}\right)$ of I- and D-LA was determined by ${ }^{1} H$ NMR endgroup analysis, as previously reported by Kricheldorf et al. ${ }^{15}$. GPC was used to determine molecular weights and molecular weight distributions $\left(\bar{M}_{\mathrm{w}} / \bar{M}_{\mathrm{f}}\right)$ of the polymers. A Waters $6000 \mathrm{~A}$ GPC apparatus equipped with three Waters $\mu$ Styragel $\left(10^{3}, 10^{4}\right.$ and $10^{5} \AA$ pore diameter) columns was used. A polymer solution in dichloromethane $(0,5 \mathrm{wt} .-\%)$ was used, and elution was performed at $25^{\circ} \mathrm{C}$ at a flow rate of 2,0 $\mathrm{ml} / \mathrm{min}$ using dichloromethane as eluent. The molecular weights were determined using a 
calibration curve for poly(I-lactide) which was prepared by an absolute method based on combined GPC and low angle laser light scattering measurements ${ }^{19}$. Specific rotations of polymers were measured at a concentration of $0,01 \mathrm{~g} / \mathrm{ml}$ in chloroform on a Perkin-Elmer 241 Polarimeter employed with sodium light and a light pass length of $10 \mathrm{~cm}$ at $25^{\circ} \mathrm{C}$. The thermal analysis of polymers was carried out with a Perkin-Elmer DSC4 differential scanning calorimeter calibrated with pure indium. The polymers were melted at $250^{\circ} \mathrm{C}$ for $5 \mathrm{~min}$, followed by quenching to room temperature, and the curves were then recorded at a heating rate of $15^{\circ} \mathrm{C} / \mathrm{min}$.

\section{Results and discussion}

In order to confirm the feasibility of the synthesis of lactide stereo-block copolymers initiated by aluminium tris(2-propanolate) (1), the polymerization of $\mathrm{L}$ - or D-lactide (LA) with various monomer/initiator mole ratios ([M]/[I]) was carried out in toluene solution at $90^{\circ} \mathrm{C}$. It was shown that poly(LA)s with isopropoxycarbonyl end-groups were obtained and no alkyloxy end-groups were detected. This indicates that the LA polymerization, initiated by the alcoholate 1 proceeds only through the acyl-oxygen cleavage of LA.

A "living" character of the polymerization initiated by 1 was confirmed by an accurate correlation between the number-average degree of polymerization $\left(\overline{\mathrm{DP}}_{n}\right)$ and the $[\mathrm{M}] /[\mathrm{I}]$ ratio at complete conversion, as shown in Fig. 1. From the slope of the line in Fig. 1, it can be seen that all of the three isopropoxy groups in the initiator molecule

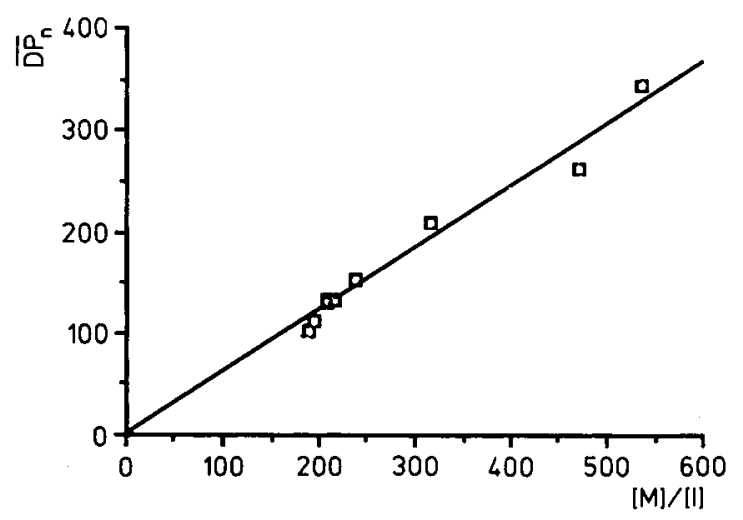

Fig. 1. Relationship between $[\mathrm{M}] /[\mathrm{I}]$ and $\overline{\mathrm{DP}}_{\mathrm{n}}$ from NMR endgroup analysis on LA polymerization by aluminium tris(2-propanolate) in toluene at $90^{\circ} \mathrm{C}$ (complete conversion)

participate in the formation of growing chains. In the previous reports, it was mentioned that the number of active centers for initiating the chain growth depends upon the nature of the solvent and the temperature ${ }^{12,13)}$. Our results (toluene at $90^{\circ} \mathrm{C}$ ) indicate that all isopropoxy groups of the initiator molecules are active enough to participate in the initiation and propagation processes. Finally, the $\overrightarrow{\mathrm{DP}}_{\mathrm{n}}$ of poly(LA)s was determined by three methods, namely, the calculation based on the $[\mathrm{M}] /[\mathrm{I}]$ ratio 
and monomer conversion, measured by ${ }^{1} \mathrm{H}$ NMR, the quantitative ${ }^{1} \mathrm{H}$ NMR endgroup analysis, and by GPC measurement. The $\overline{\mathrm{DP}}_{\mathrm{n}}$ values obtained from both the NMR endgroup analysis and the GPC measurement were consistent with those obtained from the calculations, assuming that all alkoxy groups are active. It is concluded that the LA polymerization by 1 proceeds through a "living" type of polymerization.

Specific rotations of poly(L-LA)s and poly(D-LA)s with different molecular weights were measured, and the results are summarized in Tab. 1. Poly(I-LA)s and poly(D-LA)s had the same specific rotations as previously reported values ${ }^{8}$. This result indicates that no racemization occurred during the LA polymerization with initiator 1 . Therefore, it is considered that $\mathbf{1}$ is a suitable initiator for preparing our designed diblock copolymers.

Tab. 1. Optical activities of poly(t- or D-lactide)s

\begin{tabular}{lcc}
\hline Sample & $\bar{M}_{\mathrm{n}}^{\mathrm{a})}$ & {$[\alpha]_{\mathrm{D}}^{25 \mathrm{c})}$} \\
\hline PLLA & 10000 & -153 \\
PLLA & 24600 & -155 \\
PDLA & 9700 & +155 \\
PDLA & 24900 & +157 \\
a) PLLA: poly(L-lactide); PDLA: poly(D-lactide). & \\
b) Calculated from monomer conversions. & \\
c) Specific rotation determined in chloroform at $25^{\circ} \mathrm{C}(1,0 \mathrm{~g} / \mathrm{ml})$. &
\end{tabular}

Block copolymerization of L- and D-LAs was carried out by sequential addition of a D-LA solution to a completely polymerized L-LA system. The results of the block copolymerization are listed in Tab. 2. In run no. 1 (Tab. 2) the monomer conversion in the second step (D-LA) polymerization was low, which was due to a high heterogeneity in the reaction mixture. In order to get a more homogeneous reaction, other block copolymerizations were carried out in a revised manner: when the reaction mixture became somewhat turbid during the second step polymerization, toluene corresponding to ca. 15 vol. $-\%$ of the reaction solution was added to the solution and the reaction temperature was shifted $5^{\circ} \mathrm{C}$ upwards. Although still some turbidity existed in the solution, this adjustment leads to a considerable improvement, and block copolymerizations proceeded in much higher conversions, as listed in Tab. 2.

The molecular weights of copolymers determined by the NMR endgroup analysis and the GPC measurement were in agreement with the calculated values based on the $[\mathrm{M}] /[\mathrm{I}]$ ratio and monomer conversion, according to a living polymerization mechanism. The resumption of D-LA polymerization onto a polymerized L-LA system with proportional increase of molecular weight was also ascertained by the GPC measurement. A representative result of the GPC measurement is shown in Fig. 2. An increase in molecular weight of the final product [Fig. 2(A)] from that of the 


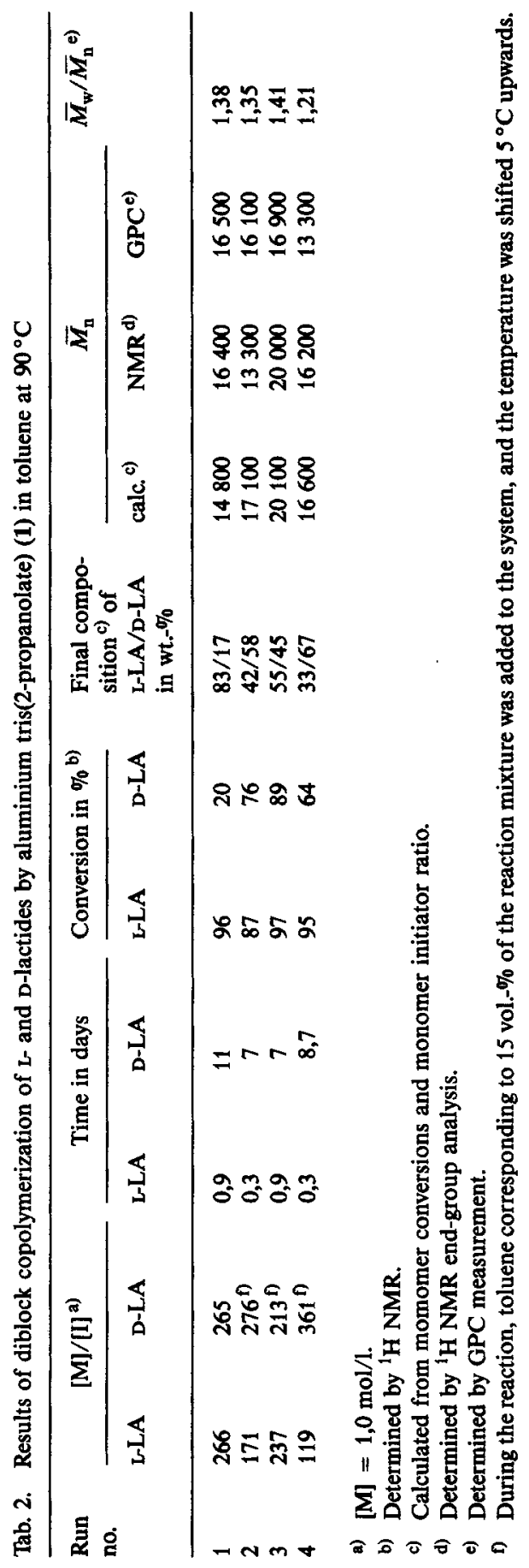




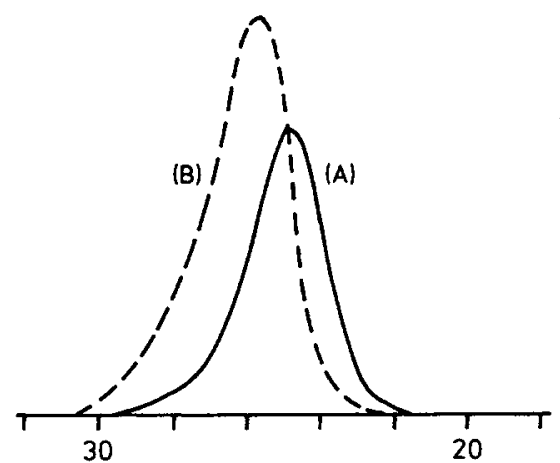

Elution volume in $\mathrm{ml}$
Fig. 2. GPC curves of a diblock copolymer of $\mathrm{L}$ and $\mathrm{D}$-LAs and the corresponding prepolymer of $\mathrm{L}-\mathrm{LA}$. (A): Block copolymer $\bar{M}_{\mathrm{n}}=16100$, $\bar{M}_{\mathrm{w}} / \bar{M}_{\mathrm{n}}=1,35 ;$ (B): prepolymer $\bar{M}_{\mathrm{n}}$ $=8600, \bar{M}_{\mathrm{w}} / \bar{M}_{\mathrm{n}}=1,44$

prepolymer [Fig. 2(B)] with a unimodal molecular weight distribution was observed, confirming the formation of block copolymers of $\mathrm{L}$ - and D-LAs. Block copolymers had $\bar{M}_{\mathrm{w}} / \bar{M}_{\mathrm{n}}$ values of around $1,2-1,4$, and these values are almost the same as those obtained with 1 or some bimetallic oxoalkoxide reported by Teyssié et al. ${ }^{13,17,18)}$. All these results indicate that additional D-LA was actually polymerized onto the polymerized L-LA chain according to a living polymerization mechanism.

Specific rotations of block copolymers were measured, and the results are summarized in Tab. 3. The specific rotations of block copolymers were found to depend upon the enantiomeric excess of $\mathrm{L}$ - or D-LA. The L-LA contents in the copolymers calculated from these values are well in the agreement with those from monomer conversions. These results indicate that no racemization occurred during the block copolymerization of $\mathrm{L}$ - and D-LAs.

It is concluded that sequential diblock copolymers of $\mathrm{L}$ - and D-lactides with different block-lengths can be successfully synthesized by a living ring-opening polymerization of $\mathrm{L}$ - and D-LAs initiated by 1 .

Tab. 3. Optical acivities of diblock copolymers of $\mathrm{z}$ - and D-lactides

\begin{tabular}{lccccc}
\hline Run no. & $\begin{array}{l}\text { L-LA content } \\
\text { in } \%\end{array}$ & $\begin{array}{l}\text { Enantiomeric } \\
\text { excess in } \%\end{array}$ & $\bar{M}_{\mathrm{n}}{ }^{\text {a) }}$ & {$[\alpha]_{\mathrm{D}}^{25 \mathrm{c})}$} & $\begin{array}{l}\text { L-LA content } \\
\text { in } \%\end{array}$ \\
\hline 1 & 83 & 66 & 16400 & -99 & 82 \\
2 & 42 & 16 & 17100 & +15 & 45 \\
3 & 55 & 10 & 20100 & $-6,5$ & 52 \\
4 & 33 & 34 & 16600 & +42 & 37
\end{tabular}

a) Calculated from monomer conversions and monomer/initiator ratio.

b) Calculated from the following equation: $=100 \% \times($ L-LA $-D-L A) /(L-L A+D-L A) ; L-L A$ or D-LA expressed in mol.

c) Specific rotation determined in $1,0 \mathrm{~g} / \mathrm{ml}$ solution in chloroform at $25^{\circ} \mathrm{C}$.

d) Calculated from specific rotation of copolymer, assuming that the specific rotations of poly(I-lactide) and poly(D-lactide) are -154 and +156 , respectively. 


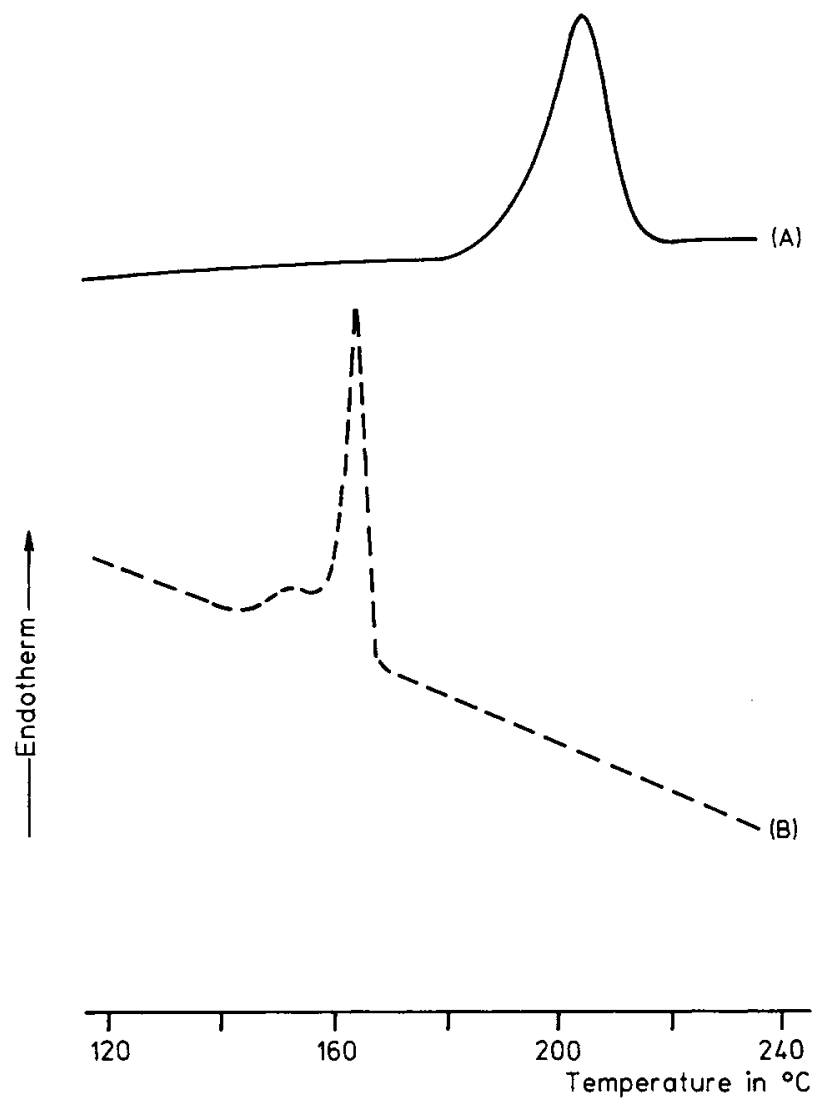

Fig. 3. DSC thermograms of a diblock-copolymer of $\mathrm{L}$ - and D-LAs and the corresponding homopolymer of L-LA. (A): Block copolymer $\bar{M}_{\mathrm{n}}=20100$, L-LA content $=55 \%$; (B): homopolymer $\bar{M}_{\mathrm{n}}=10000$

Quite recently it was found that these block copolymers have much higher melting temperatures and lower solubilities than the corresponding homopolymers. Representative DSC thermograms of homopolymer, poly(I-LA), and a block copolymer are shown in Fig. 3. The block copolymer shows a melting temperature around $205^{\circ} \mathrm{C}$, which is approximately $40^{\circ} \mathrm{C}$ higher than that of poly(L-LA).

This result provides strong evidence for the stereocomplex formation between poly(L-LA) and poly(D-LA) chains in the copolymer. Detailed characterization and properties of these block copolymers will be reported in a forthcoming paper.

Nobuhiko Yui wishes to thank the Department of Chemical Technology, University of Twente, the Netherlands, for its research fellowship. The authors are grateful to Hetty Geerts for performing the GPC measurements. 
1) R. K. Kulkarni, E. G. Moore, A. F. Hegyeli, F. Leonard, J. Biomed. Mater. Res. 5, 169 (1971)

2) S. W. Kim, R. V. Petersen, J. Feijen, "Polymeric Drug Delivery Systems", in "Drug Design", vol. 10, ed. by E. J. Ariens, Academic Press, New York 1980, p. 193

3) D. A. Wood, Int. J. Pharm. 7, 1 (1980)

4) B. Eling, S. Gogolewski, A. J. Pennings, Polymer 23, 1587 (1982)

5) J. Heller, CRC Crit. Rev. Ther. Drug Carrier Syst. 1, 39 (1985)

9) S. Y. Jeoung, S. W. Kim, Arch. Pharm. Res. 9, 63 (1986)

7) S. J. Holland, B. J. Tighe, P. L. Gould, J. Controlled Release 4, 155 (1986)

8) F. Chabor, M. Vert, S. Chapelle, P. Granger, Polymer 24, 53 (1983)

9) Y. Ikada, K. Jamshidi, H. Tsuji, S.-H. Hyon, Macromolecules 20, 904 (1987)

10) US. 4719246 (1988), E. I. Du Pont de Nemours and Co., invs.: J. R. Murdock, G. L. Loomis; Chem. Abstr. 108, 132811 y (1988)

11) D. Grenier, R. E. Prud'homme, J. Polym. Sci., Polym. Phys. Ed. 22, 577 (1984)

12) T. Ouhadi, C. Stevens, P. Teyssié, Makromol. Chem., Suppl. 1, 191 (1975)

13) J.-M. Vion, R. Jérôme, P. Teyssié, M. Aubin, R. E. Prud'homme, Macromolecules 19, 1828 (1986)

14) H. R. Kricheldorf, T. Mang, J. M. Jonté, Macromolecules 17, 2173 (1984)

15) H. R. Kricheldorf, M. Berl, N. Scharnagl, Macromolecules 21, 286 (1988)

16) M. J. D. Eenink, $P h$. D. Thesis, University of Twente, Enschede, The Netherlands, 1987

17) P. Teyssié, T. Ouhadi, J. P. Bioul, "New Prospects in Homogeneous Ring-opening Polymerization of Heterocyclic Monomers", in "Int. Rev. Sci. Phys. Chem., Ser.", vol. 8, Butterworth, London 1975, p. 191

18) A. Hamitou, R. Jerome, P. Teyssié, J. Polym. Sci., Polym. Chem. Ed. 15, 1035 (1977) 